\title{
Altered Pulmonary Venous Flow Pattern in Young Adults With Atrial Septal Defect
}

\author{
Mozhgan Parsaee ${ }^{1}$, Sedigheh Saedi ${ }^{2,{ }^{*}}$, Nahid Salehi ${ }^{3}$, Tahereh \\ Saedi $^{2}$ \\ ${ }^{1}$ Echocardiography Research Center, Rajaie Cardiovascular Medical and Research \\ Center, Iran University of Medical Sciences, Tehran, Iran \\ ${ }^{2}$ Rajaie Cardiovascular Medical and Research Center, Iran University of Medical \\ Sciences, Tehran, Iran \\ ${ }^{3}$ Departement of Cardiology, Kermanshah University of Medical Sciences, \\ Kermanshah, Iran \\ ${ }^{*}$ Corresponding author: Sedigheh Saedi, Niayesh Highway, adjacent to Mellat Park, \\ Rajaie Cardiovascular Medical and Research Center, Iran University of Medical \\ Sciences, Tehran, Iran. Tel: +98-2123922003,E-mail: sedsaedi@gmail.com
}

DOI: $10.20286 / \mathrm{ijcp}-010206$

Submited: 05.28.2015

Accepted: 07.09.2015

Keywords:

Pulmonary Veins

Echocardiography

(C) 2016. International Journal of Cardiovascular Practice.
Heart Septal Defects, Atrial

\begin{abstract}
Introduction: Atrial septal defect (ASD) is a common congenital heart disease and causes left-to-right shunting and significant right ventricular (RV) volume overload. The goal of this study was to evaluate the influence of these hemodynamic changes on pulmonary venous flow pattern in young adults.

Methods: Complete echocardiographic examination was performed in a group of 40 young adults (aged < 40 years) who had secundum type ASD and was compared to 40 age-matched individuals in control group who had no cardiac abnormality. Systolic and diastolic flow velocities in pulmonary veins (PV), superior vena cava (SVC), inferior vena cava (IVC) and RV functional parameters were recorded and evaluated.

Results: As opposed to healthy young individuals who showed distinct $S$ and D waves with diastolic predominance in pulmonary vein Doppler, in patients with ASD a continuous flow with increased systolic peak that began in systole and continued to the late diastole was observed. The RV systolic function increased compared to the control group.

Conclusions: In patients with ASD, the pattern of pulmonary veins flow transforms into a single continuous antegrade wave with systolic dominance due to persistent shunting of left atrial blood in to right heart chambers as well as increased RV pump function on pulmonary vein (by means of ASD), SVC and IVC, and could be used as a screening method for the presence of secundum type ASDs in young adults.
\end{abstract}

\section{INTRODUCTION}

Atrial septal defect (ASD) is a commonly encountered yet easily missed congenital heart defect in young adults. ASDs are abnormally persistent connections between the left and right atriums with resultant left-to-right shunting of blood and RV volume overload. The most common subtype is the ostium secundum type [1].

The pulmonary venous flow pattern is related and complementary to mitral inflow velocities for the assessment of diastolic dysfunction. It is recorded 1-2 mm inside the orifice of pulmonary veins by transthoracic and transesophageal echocardiography and has three main components: The $S$ wave which is recorded during the forward flow of blood into the left atrium at the time of ventricular systole, D wave or diastolic wave which is contemporary to ventricular diastole, and atrial reversal wave during atrial systole. However, the pulmonary venous flow pattern has proven to change by aging. In healthy children and young adults, there is a pattern

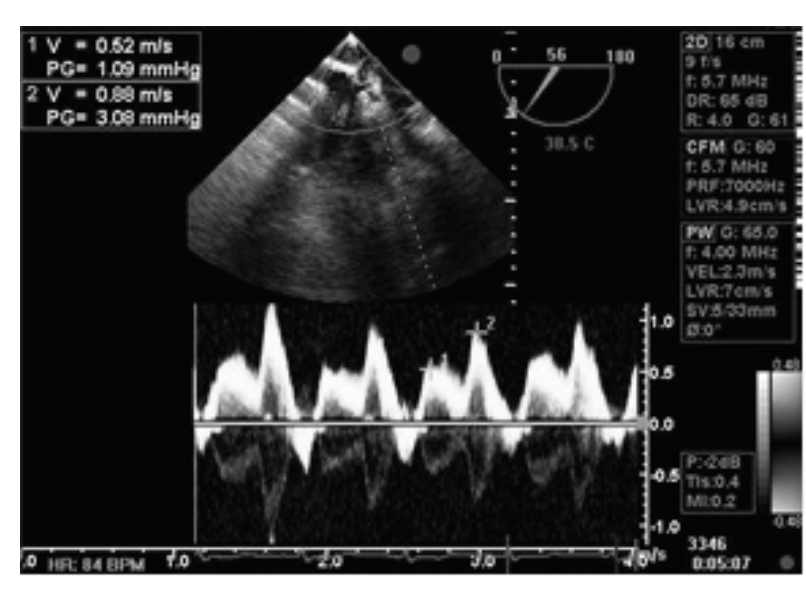

Figure 1: A, Pulmonary Flow Pattern of a Healthy Young Woman Showing Systolic or S Wave B, Less Than Diastolic or D Wave 
of diastolic predominance and resultant S/D ratio $<1$ (Fig $1)$. As individuals age, the systolic component becomes more dominant, the atrial reversal increases and the S/D becomes $>1$ after the age of 50 [2-4].

In patients with ASD, there might be alterations in the pulmonary venous flow pattern due to the communication with the right atrium. In this study, we sought to investigate the changes in pulmonary vein flow velocities and pattern in young patients with secundum type ASD.

\section{METHODS}

\section{Study Group}

In this study, 40 young adults (age $<40$ years) with ASD but without any other cardiac disorder including significant valvular heart disease or cardiomyopathies were enrolled. The control group consisted of 40 healthy, age-matched normal young adults who were free from any cardiac anomalies. All the patients in the ASD group underwent a complete transthoracic (TTE) and transesophageal echocardiography (TEE) including M-mode, two-dimensional, spectral, color and tissue Doppler evaluations. The control group was evaluated by TTE. Informed consent was obtained from all the participants and the hospital ethics committee approved the study protocol. Mitral inflow Doppler findings including peak E and A-wave velocities were recorded in four chamber TTE view. Pulmonary venous Doppler data including peak $S$ and $D$ wave velocities and atrial reversal wave were measured in mid-esophageal TEE views by placing the sample volume in all the right and left pulmonary veins and in TTE apical four chamber view. The right ventricular systolic function was evaluated by tissue Doppler imaging (systolic annular velocity) and tricuspid annular plane systolic excursion (TAPSE) methods.

\section{Statistical Analysis}

Statistical analysis was performed with SPSS 15 for Windows (SPSS Inc., Chicago, Illinois). All the values were expressed as mean \pm SEM. Normality of distribution was assessed through the use of Kolmogorov-Smirnov test. Categorical values were compared by chi-squared test or Fisher's exact test. To compare the mean variables between two groups, an independent t-test or Mann-Whitney $U$ test was used. $\mathrm{P}<0.05$ was considered to be significant.

\section{RESULTS}

The mean age in the ASD group was $28.7 \pm 7.6$ years old with the youngest patient being 16 years old and the oldest 40 , and $27.6 \pm 9.1$ years old in the control group. The mean ASD size was $2.2 \pm 0.67 \mathrm{~cm}$ with mean echocardiographically estimated $\mathrm{Qp} / \mathrm{Qs}$ of $2.6 \pm 0.8$. The mean pulmonary artery systolic pressure (SPAP) was $35.4 \pm 8$ in the ASD group and $26 \pm 6$ in the control group.

The mean peak $S$ velocity and peak $D$ velocity of right upper pulmonary vein in the normal control group were 45.9 and $60.9(\mathrm{~S} / \mathrm{D}<1)$, respectively. A mainly continuous antegrade wave was recorded in the ASD group with the mean peak $S$ velocity and peak $D$ velocity being 60.2 and 51.5 in the right pulmonary veins and 57.98 and 49.2 in the left pulmonary veins (i.e. increased systolic peak), respectively (Tables 1 and 2) (Figs 2 and 3 ).

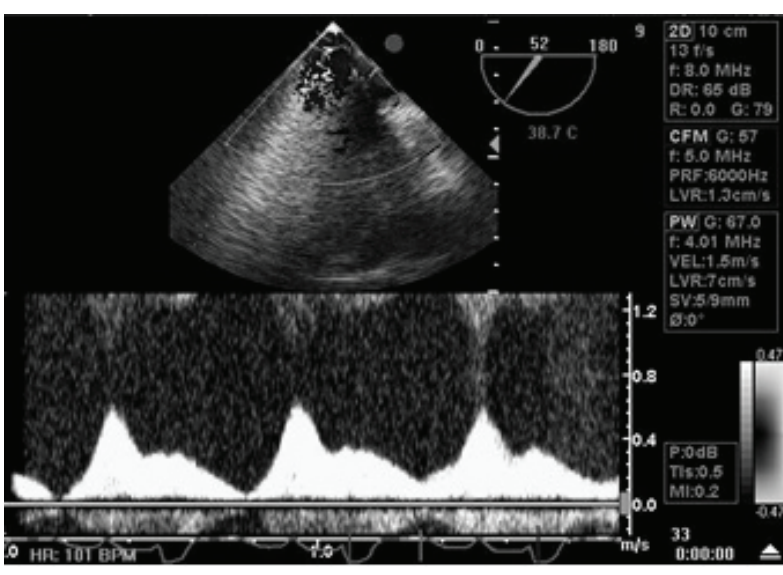

Figure 2: Transesophageal Echocardiography in a Young Male Patient Who Was Diagnosed to Have a Large Secundum Type Atrial Septal Defect and Showing a Continuous Wave From the Start of Systole to End Diastole With a S > D Pattern

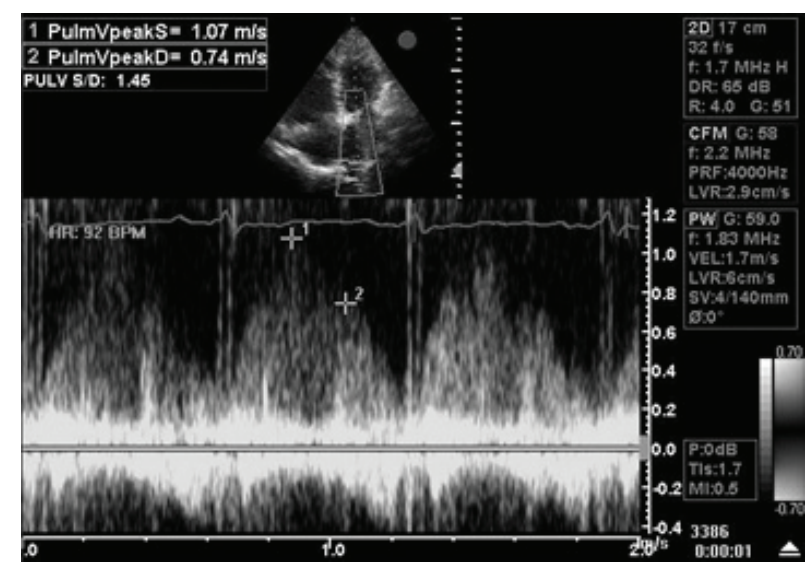

Figure 3: Transthoracic Echocardiographic Pulmonary Venous Flow in a patient With Atrial Septal Defect Also Showing a Continuous Antegrade Wave With S > D Pattern

There was S > D in superior vena cava (95\%), inferior vena cava $(87.5 \%)$ and ASD flow (85\%) in the latter group with no statistically significant difference from the normal control group for the SVC and IVC flow, although the mean recorded systolic velocity of SVC was greater in the ASD group.

In the ASD group, 31 patients $(77.5 \%)$ had $S>D$ in the right pulmonary veins flow and 34 patients $(85 \%)$ had $S>D$ in the left pulmonary veins, while in the normal control group 38 individuals (95\%) had S < D in right pulmonary vein flows. The difference between the two groups was statistically significant $(\mathrm{P}<0.005)$. In the ASD group, those with $\mathrm{S}>\mathrm{D}$ flow velocities in pulmonary veins had a larger size of ASD (mean $=2.2 \pm 0.7 \mathrm{~cm}$ vs. $1.7 \pm 0.8 \mathrm{~cm}$ in those with $\mathrm{S}<\mathrm{D}$ ).

There was no statistically significant difference in the trans-mitral $\mathrm{E}$ and $\mathrm{A}$-wave velocities or deceleration time and in E/E' between ASD and control groups. The LV diastolic function was normal in all the patients.

As for the RV functional parameters, the mean tricuspid annular plane systolic excursion (TAPSE) in the ASD group was 26.73 compared to 25.2 in the control group. Also, the mean systolic velocity $(\mathrm{Sm})$ was 14.13 in the ASD group 
and 13.6 in the control group. Although both parameters increased in the ASD group, they did not reach statistical significance. Patient and echocardiographic characteristics of the ASD group are shown in Table 1.

\begin{tabular}{|lccc|}
\hline \multicolumn{4}{|l}{ Table 1: Patient and Echocardiographic } \\
Group
\end{tabular}

ASD, atrial septal defect; BSA, body surface Area; D, diastolic velocity; IVC, inferior vena cava; LTPV, left pulmonary veins; MV-A, mitral late diastolic inflow velocity; MV-E, mitral early diastolic inflow velocity; PAP, pulmonary artery systolic pressure; RTPV, right pulmonary veins; $S$, systolic velocity; $\mathrm{Sm}$, systolic right ventricular velocity; SVC, superior vena cava; TAPSE, tricuspid annular plane systolic excursion.

\begin{tabular}{|c|c|c|c|}
\hline & Number (\%) & Valid Percent & Cumulative Percent \\
\hline \multicolumn{4}{|l|}{ RUPV } \\
\hline $\mathrm{S}>\mathrm{D}$ & $31(77.5)$ & 77.5 & 77.5 \\
\hline $\mathrm{D}>\mathrm{S}$ & $9(22.5)$ & 22.5 & 100 \\
\hline Total & $40(100)$ & 100 & \\
\hline \multicolumn{4}{|l|}{ LUPV } \\
\hline $\mathrm{S}>\mathrm{D}$ & $34(85)$ & 85 & 85 \\
\hline $\mathrm{D}>\mathrm{S}$ & $6(15)$ & 15 & 100 \\
\hline Total & $40(100)$ & 100 & \\
\hline
\end{tabular}
vein.

\section{DISCUSSION}

In the present study, we aimed to evaluate the pattern of pulmonary vein flow in a group of older patients with previously un-intervened secundum type ASD (aged over 16). Schoroh AM et al. in their study of 74 patients with ASD aged under 15 , showed that there were increased systolic velocities and decreased diastolic pattern velocities in pulmonary veins of patients with left-to-right atrial level shunting [2]. As shown in our study, hemodynamically significant ASDs change the pulmonary venous flow pattern towards merging of systolic and diastolic waves, with systolic dominance in young adults and an S/D ratio of $>1$ in measurements. We also found a statistically significant correlation between ASD size and pattern of pulmonary vein flow. The larger the ASD size and the amount of left-to-right shunting as measured by Qp/Qs, the more prominent this pattern becomes. Studies have reported a decrease in S/D ratio after successful closure of the ASD [5-9].

The pulmonary vein flow pattern is formed based on the pressure difference between the PVs and left atrium. In patients with a large ASD, there is a significant left-to-right shunting of the blood. This leads to decreased effect of the left heart pressures on the PV flow pattern, as in this setting only a part of the PV blood goes to the LA and mitral valve, whereas the rest enters the more compliant right heart chambers. Therefore, the PV flow pattern is mostly shaped by left-to-right shunting, leading to a characteristic antegrade continuous wave with systolic dominance in PV Doppler as a result of blood runoff into RA via ASD [4].

As mentioned earlier, there was a statistically non-significant increase in RV systolic function parameters in the ASD group versus the control group, which is in accordance with findings of previous studies showing increased contractile function of the right ventricle and displacement of the tricuspid annular plane in patients with ASD [2]. These changes in function might be explained by the RV volume overload (Frank-Starling law) followed by secondarily enhanced suctioning of the blood that enters the heart through pulmonary veins, which connect to RA and RV in the presence of ASD. When RV systolic dysfunction happens, the systolic pulling of blood decreases [2, 10-12].

The increased systolic velocities in SVC, IVC and the ASD itself could be explained in a similar manner and by the same mechanism. Increased RV contractility and enhanced emptying of the right atrium cause a vacuuming effect on the above mentioned structures $[2,4]$.

These changes do not seem to affect mitral $\mathrm{E}$ and $\mathrm{A}$ wave velocities. However, this is rather difficult to precisely evaluate due to different hemodynamic effects on mitral velocities $[2,4]$. In this study, we used TEE for accurate recording of $S$ and D wave velocities in the ASD group. A limitation of our study was impossibility to use TEE in the healthy control group due to the semi-invasive nature of this procedure. However, as the flow of most pulmonary veins can be easily obtained by transthoracic echocardiography and since measurements of pulmonary vein flows were comparable between TTE and TEE in our study in the ASD group, we believe that transthoracic echocardiography could provide important screening information regarding the presence and hemodynamic significance of ASDs in routine clinical practice. Other studies have also reported valid and reliable measurements of pulmonary vein flow by TTE $[3,13]$.

In summary the present study aimed to determine pulmonary venous flow abnormalities in patients with ASD below the age of 40 . The measurement of pulmonary venous flow by TTE was feasible and accurate compared to TEE findings. ASD modifies the pulmonary venous flow towards a merged and continuous antegrade wave with prominence of systolic 
time and peak. Enhanced RV systolic function, especially by means of annular plane displacement due to the volume overload, could account for these alterations. The changes could help to identify a missed ASD in the young population and should not be mistaken for early and subclinical left ventricular diastolic dysfunction.

\section{Study Limitations}

The healthy control group did not undergo TEE due to its semi-invasive nature, but studies have shown that the patterns of pulmonary vein flow, especially that measured in the right upper pulmonary vein correlate well with TEE measurements [3].

\section{CONFLICTS OF INTEREST}

There is no conflict of interest.

\section{REFERENCES}

1. Webb GD, Smallhorn JF, Therrien J. Congenital Heart Disease In: Braunwald heart disease 10th ed2015.

2. Schroh AM, Laghezza LB, Dominguez PJ, Brandan V, Nento DE, Alvarez E, et al. Pattern of pulmonary venous flow in patients with ostium secundum atrial septal defect. Eur J Echocardiogr. 2009;10(2):244-9. DOI: 10.1093 /ejechocard/jen213 PMID: 18682408

3. Tabata T, Thomas JD, Klein AL. Pulmonary venous flow by doppler echocardiography: revisited 12 years later. J Am Coll Cardiol. 2003;41(8):1243-50. PMID: 12706916

4. Fadel BM, Mohty D, Aldawood W, Dahdouh Z, Di Salvo G. Spectral Doppler interrogation of the pulmonary veins in atrial septal defect. Echocardiography. 2015;32(6):1027-9. DOI: 10.1111/echo.12892 PMID: 25611569
5. Gomez CA, Ludomirsky A, Ensing GJ, Rocchini AP. Effect of acute changes in load on left ventricular diastolic function during device closure of atrial septal defects. Am J Cardiol. 2005;95(5):686-8. DOI 10.1016/j.amjcard.2004.10.052 PMID: 15721123

6. Saric M, Applebaum RM, Phoon CK, Katz ES, Goldstein SA, Tunick PA, et al. Pulmonary venous flow in large, uncomplicated atrial septal defect. J Am Soc Echocardiogr. 2001;14(5):386-90. PMID: 11337684

7. Lin WW, Fu YC, Jan SL, Wang KY, Ho HC, Lin FY, et al. Immediate change in pulmonary venous flow pattern after deployment of occluder device for atrial septal defect. Echocardiography. 2009;26(4):452-8 DOI: 10.1111/j.1540-8175.2008.00835.x PMID: 19382945

8. Chockalingam A, Dass S, Alagesan R, Muthukumar D, Rajasekar MA, Subramaniam T, et al. Role of transthoracic Doppler pulmonary venous flow pattern in large atrial septal defects. Echocardiography. 2005;22(1):9-13. DOI: $10.1111 / j .0742-2822.2005 .03171 . x$ PMID 15660681

9. Pauliks LB, Chan KC, Chang D, Kirby SK, Logan L, DeGroff CG, et al. Regional myocardial velocities and isovolumic contraction acceleration before and after device closure of atrial septal defects: a color tissue Doppler study. Am Heart J. 2005;150(2):294-301. DOI: 10.1016/j. ahj.2004.09.052 PMID: 16086934

10. Lytrivi ID, Lai WW, Ko HH, Nielsen JC, Parness IA, Srivastava S. Color Doppler tissue imaging for evaluation of right ventricular systolic function in patients with congenital heart disease. J Am Soc Echocardiogr 2005;18(10):1099-104. DOI: 10.1016/j.echo.2005.03.029 PMID $\underline{16198888}$

11. Frommelt PC, Ballweg JA, Whitstone BN, Frommelt MA. Usefulness of Doppler tissue imaging analysis of tricuspid annular motion for determination of right ventricular function in normal infants and children. Am J Cardio. 2002;89(5):610-3. DOI: 10.1016/S0002 9149(01)02308-6

12. PMID: 11867053

13. Yilmiz M. Pulmonary venous flow characteristics in ostium secundum type Atrial Septal Defect. Türk Kardiyol Dem Ars. 2003;31:426-31.

14. Lam YY, Fang F, Yip GW, Li ZA, Yang Y, Yu CM. New pulmonary vein Doppler echocardiographic index predicts significant interatrial shunting in secundum atrial septal defect. Int J Cardiol. 2012;160(1):59-65 DOI: 10.1016/i.ijcard.2011.03.031 PMID: 21477873 